\title{
A Comparative Study of Fuzzy and Intuitionistic Fuzzy Techniques in a Knowledge based Decision Support
}

\author{
Asma R Shora \\ Research Scholar \\ Jamia Hamdard University
}

\author{
M Afshar Alam \\ Professor \\ Jamia Hamdard University
}

\author{
Ranjit Biswas \\ Head of Department CS \\ Jamia Hamdard University
}

\begin{abstract}
Through this paper we present the implementation of fuzzy decision making in case of a medical diagnostic system and compare the results with those of the Intuitionistic fuzzy decision making technique. Both the approaches are used to solve multi-criteria, complex decision making problems. Intuitionistic fuzzy differs from the fuzzy approach as it adds indeterminacy and imprecision to the fuzzy technology. Let us consider a simple diagnostic system where we diagnose the causes of obesity. Patients are treated for obesity according to the type of obesity they have or let us say the cause of their obesity. Obesity can be lifestyle related or it can be a pathological or genetic disorder. Both fuzzy and Intuitionistic fuzzy techniques will determine the cause of obesity. It is for us to decide which one is the efficient way to do it.
\end{abstract}

\section{Keywords}

decision support, knowledge base, Fuzzy, Intuitionistic Fuzzy.

\section{INTRODUCTION}

The Intuitionistic fuzzy decision support system has been already implemented in our previous work [1] and our aim is to use Fuzzy decision making so as to demonstrate and compare the two techniques. IF decision making got a little attention in the past [5]. A considerable amount of work has been done on the IF expert systems $[3,8]$ as well. We are diagnosing the causes of obesity. The same case study [1] is used again so as to make the comparison clear. We have a medical knowledge base which comprises of both crisp and fuzzy values for fuzzy approach and for the Intuitionistic fuzzy approach, we have IF (Intuitionistic fuzzy) values in place of fuzzy data. Our first step will be quantization of symptoms. Symptoms and their extent is usually expressed, observed and recorded in the form of natural linguistic variables e.g. severe pain, little drowsiness, etc. These have to be converted and examined in the form of numbers when automation comes into play. [11] already brought forth the need of computing using linguistic variables. The problem that arises here is to map human complex logic into a set of numbers. Crisp sets have a limitation that they can be used to represent binary logic or for that matter "two state decisions". This hinders the automation process. Thus, fuzzy and intuitionistic fuzzy sets come to our rescue and we can represent multi-criteria decisions using set based logic. Second step comprises filtering out those patients, who are suffering from disorder induced obesity. For this purpose, we will use fuzzy sets and compare the results with the previous technique.

\section{PRELIMINARIES}

Fuzzy logic is a form of logic that involves variables with more than two possible values i.e. many-valued logic or probabilistic logic; Vagueness is involved while dealing with such scenarios where approximate values are being used instead of exact values. While as the traditional logic theory, where binary sets have two-valued logic, true or false, fuzzy logic variables may have a truth value that ranges in degree between 0 and 1. Fuzzy logic handles the concept of partial truth. The truth values in this case lie somewhere between completely true and completely false. Also, specific functions are used to represent the the range of values for the unconventional linguistic variables.

Intuitionistic Fuzzy Sets were introduced by Krassimir Atanasov [7]. It was introduced as a generalised case for the existing Fuzzy Sets. IFS adds a third dimension to the existing fuzzy set theory i.e. Non-Membership (Indeterminacy/ hesitation). . Many attempts have been put forward to handle the imprecision in the real world logic so far. The concept of uncertain sets [10] is also closely related to IF sets, the way the operations and functions have been defined but IF sets seem to be better established.

\subsection{Definition I}

Let a set $\mathrm{E}$ be fixed. An IFS $\mathrm{A}$ in $\mathrm{E}$ is an object of the following form:

$\mathrm{A}=\left\{\left(\mathrm{x}, \mu_{\mathrm{A}}(\mathrm{x}), v_{\mathrm{A}}(\mathrm{x})\right) \mathrm{x} € \mathrm{E}\right\}$, When $v_{\mathrm{A}}(\mathrm{x})=1-\mu_{\mathrm{A}}(\mathrm{x})$ for all $\mathrm{x} € \mathrm{E}$ is ordinary fuzzy set.

In addition, for each IFS A in $\mathrm{E}, \pi_{\mathrm{A}}(\mathrm{x})=1-\mu_{\mathrm{x}}-v_{\mathrm{x}}$, where $\pi_{\mathrm{A}}$ (x) is called the degree of indeterminacy of $\mathrm{x}$ to $\mathrm{A}$, or called the degree of hesitancy of $\mathrm{x}$ to A.

\subsection{Definition II}

As an intuitionistic fuzzy set is a generalization of fuzzy set, Buckles and Petry [9] defined fuzzy database as a generalization of classical database.

A fuzzy relational database is defined as set of relations where each relation is a set of tuple. If $t_{i}$ represents the $i$-th tuple it has form $\left(\mathrm{d}_{\mathrm{i} 1}, \mathrm{~d}_{\mathrm{i} 2}, \ldots \ldots \ldots, \mathrm{d}_{\mathrm{im}}\right)$. In a classical relational database each component $\mathrm{d}_{\mathrm{ij}}$, of the tuple is an element of the corresponding scalar domain $\mathrm{Dj}$ i.e. dij belongs to $\mathrm{Dj}$. But in case of fuzzy relational database, the element of the tuple consists of either singleton or crisp subset of the scalar domain. There have been several advancements in the Fuzzy relational Database models. [6] Discusses the fuzzy ER (entity-relationship) model and fuzzy relational databases (data representation, queries, and design). 
An intuitionistic fuzzy database is a set of relation where each pair of such relation $\mathrm{R}$ is a subset of the cross product: $2 \mathrm{D} 1 \times$ $2 \mathrm{D} 2 \times \ldots \ldots .2 \mathrm{Dm}$, where $2 \mathrm{Di}=\mathrm{P}(\mathrm{Di})$ and $\mathrm{P}(\mathrm{Di})$ is the power set of $\mathrm{Di}$, here $\mathrm{R}$ is called the intuitionistic fuzzy database relation.

\section{CASE STUDY}

We use the same case study [1] for the Fuzzy chapter as well. Patients suffering from obesity can have simple lifestyle related obesity or disorder induced obesity. For the sake of convenience and understanding, we have chosen two disorders with only one symptom (other than obesity) for each. i.e. Metabolic and Cushing's syndrome with Insulin resistance and skin streaking as symptoms respectively. The data we are going to use is the same as that from the previous study except for the way of representing the same will differ.

TABLE 1: PATIENTS_SYMPTOMS

\begin{tabular}{|l|l|l|}
\hline Patient & $\begin{array}{l}\text { Insulin } \\
\text { Resistance }\end{array}$ & Skin Streaks \\
Joe & low & Mild \\
Ben & high & moderate \\
Isabella & absent & mild \\
Jacob & absent & absent \\
Ted & absent & severe \\
Calvin & low & mild \\
\hline
\end{tabular}

\section{FUZZY METHOD}

The fuzzy versions of the Intuitionistic fuzzy tolerance relations for symptoms used previously [1] are as follows:

TABLE 2: SKIN STREAKS (fuzzy)

\begin{tabular}{|l|l|l|l|l|}
\hline $\begin{array}{l}\text { Skin } \\
\text { Streaks }\end{array}$ & Mild & Moderate & Severe & Absent \\
\hline Mild & $(1.0,0.0)$ & $(0.8,0.2)$ & $(0.9,0.1)$ & $(0.2,0.8)$ \\
Moderate & $(0.8,0.2)$ & $(1.0,0.0)$ & $(0.7,0.3)$ & $(0.4,0.6)$ \\
Severe & $(0.9,0.1)$ & $(0.7,0.3)$ & $(1.0,0.0)$ & $(0.77,0.2$ \\
Absent & $(0.2,0.8)$ & $(0.4,0.6)$ & $(0.77,0.23)$ & $3)$ \\
& & & & $(1.0,0.0)$ \\
\hline
\end{tabular}

TABLE 3: INSULIN RESISTANCE (fuzzy)

\begin{tabular}{|l|l|l|l|l|}
\hline $\begin{array}{l}\text { Insulin } \\
\text { resistance }\end{array}$ & Absent & Low & Medium & High \\
\hline Absent & $(1.0,0.0)$ & $(0.9,0.1)$ & $(0.85,0.15)$ & $(0.1,0.9)$ \\
Low & $(0.85,0.15)$ & $(1.0,0.0)$ & $(0.7,0.3)$ & $(0.3,0.7)$ \\
Medium & $(0.9,0.1)$ & $(0.7,0.3)$ & $(1.0,0.0)$ & $(0.7,0.3)$ \\
High & $(0.1,0.8)$ & $(0.3,0.7)$ & $(0.7,0.3)$ & $(1.0,0.0)$ \\
\hline
\end{tabular}

The code will be same except for the class attributes will include only membership value.

Sample:

Class symptom_fuzzy

\{

Private:

Char extent[20];

double mv; // (presence/absence/ extent)

public :

symptom_fuzzy (char ext[], double mem)

if $(\operatorname{mem}<=1)$

strcpy(extent, ext);

$\mathrm{mv}=\mathrm{mem}$$$
\}
$$$$
\}
$$

else

// return error

\};

\section{Quantizing Symptoms:}

Since fuzzy logic is involved, we can only store the membership value (presence/absence/extent) of a symptom. Hence our data will look like this: 
TABLE 4 : QUANTIZED PATIENT_SYMPTOMS

\begin{tabular}{|l|l|l|l|l|}
\hline \multirow{2}{*}{ Patient } & \multicolumn{2}{|c|}{ Insulin Resistance } & \multicolumn{2}{l|}{ Skin Streaks } \\
\cline { 2 - 5 } & FUZZY & Int. FUZZY & FUZZY & $\begin{array}{l}\text { Int. } \\
\text { FUZZY }\end{array}$ \\
Joe & 0.2 & $(0.2,0.7)$ & 0.6 & $(0.3,0.7)$ \\
Ben & 0.9 & $(0.9,0.1)$ & 0.2 & $(0.2,0.7)$ \\
Isabella & 0.0 & $(0.0,1.0)$ & 0.0 & $(0.0,1.0)$ \\
Jacob & 0.0 & $(0.0,1.0)$ & 0.0 & $(0.0,1.0)$ \\
Ted & 0.0 & $(0.0,1.0)$ & 1.0 & $(1.0,0.0)$ \\
Calvin & 0.3 & $(0.3,0.0)$ & 0.0 & $(0.0,1.0)$ \\
\hline
\end{tabular}

\section{Comparison}

Considering the record of the patient named Calvin, we have:

Membership value for Insulin resistance (Fuzzy and Intuitionistic Fuzzy) $=\mathbf{0 . 3}$

Non-Membership value for Insulin resistance (Fuzzy) $=(1-$ $0.3)=\mathbf{0 . 7}$

Non- Membership value for Insulin resistance (Intuitionistic Fuzzy) $=\mathbf{0 . 0}$

It indicates that there was a hesitation level in the actual value. Clearly, the chances of Calvin not having Insulin resistance is $\mathbf{0 . 0}$ (Int. Fuzzy), which means we cannot rule out the possibility of having Insulin resistance according to the Intuitionistic Fuzzy decision support. While as, the Fuzzy

\section{Results using Intuitionistic Fuzzy approach} (same data):

Fig 1: Simple Obesity (IF)

Turbo $C++$ IDE

patients with simple obesity

Sara

Is abe 1 a

Jacob

Calvin decision support treats the possibility of not having (nonmembership value of) Insulin resistance as $\mathbf{0 . 7}$.

Fig 2: Disorders (IF)

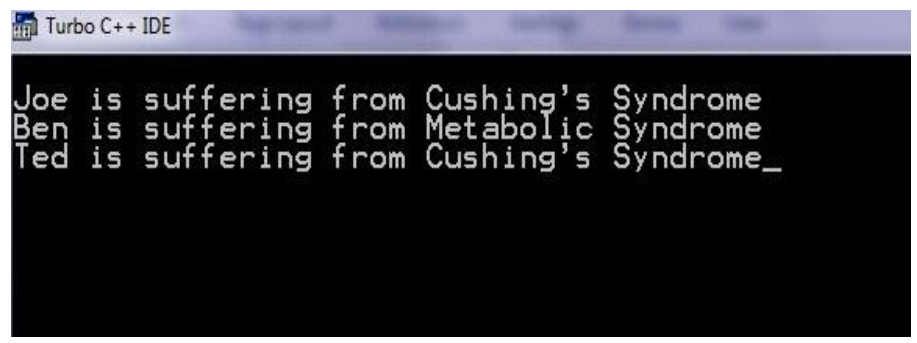

Results using Fuzzy approach (same data): Fig 3: Simple Obesity (Fuzzy)

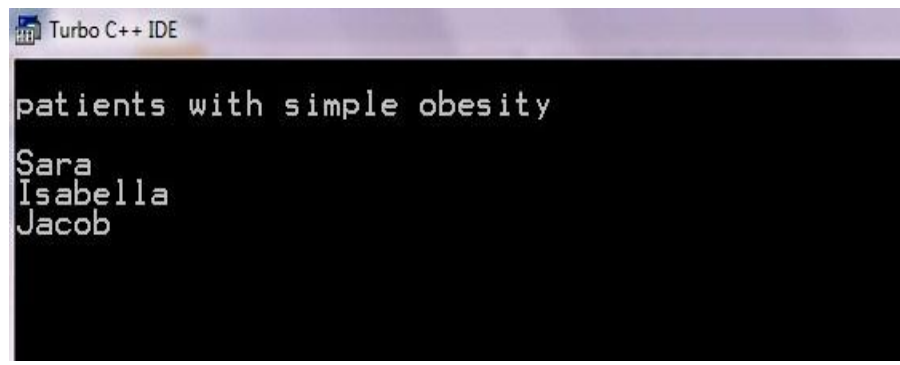

Fig 4: Disorders (Fuzzy)

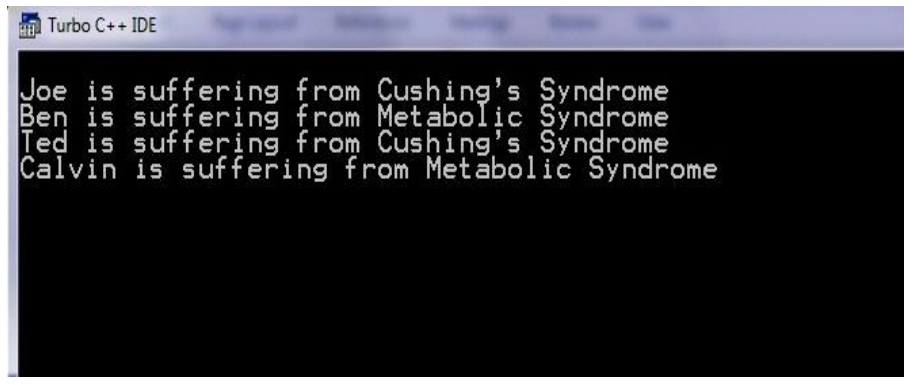

\section{Conclusion}

We compared the approaches over the same set of data and the inference that we could draw was Fuzzy and Intuitionistic Fuzzy approaches are both powerful approaches, when it comes to Multi criteria decision making. Fuzzy decision making fails only when the decisions have an uncertainty or a hesitation factor associated with them. Some studies have already shown how Intuitionistic Fuzzy problem solving can prove to have an edge over Fuzzy problem solving [2]. Fuzzy sets fail to represent information pertaining to human logic , which is vague and uncertain in nature[4]. Fuzzy sets are a specialization of Intuitionistic Fuzzy sets [7]. In our case, Fuzzy decision making approach can be used as a special case when the uncertainty / hesitation equals zero and when it is more than zero, Intuitionistic Fuzzy will be the preferred approach. 
The future scope of both fuzzy and Intuitionistic Fuzzy decision making is tremendous as the current systems rely on deterministic approach of programming. There are no set rules followed every time the system executes. In fact, new ones are employed each time depending on the criteria requirements thus making the programming more flexible and dynamic in nature. Multi - criteria decision making is used instead of binary logic. Thus making the technology look more futuristic.

\section{REFERENCES}

[1] Asma R Shora, Afshar Alam, Tamanna Sidiqui, (2012) Knowledge-driven Intuitionistic Fuzzy Decision Support for finding out the causes of Obesity, IJCSE Volume 4 Issue 3

[2] Fatih Emre Boran (2011) An integrated intuitionistic fuzzy multicriteria decision making method for facility location selection, Mathematical and Computational Applications, (Vol. 16, No. 2, pp. 487-496, 2011.).

[3] Chris Cornelis, E.E. Kerre(2001). Inclusion based approximate reasoning. In lecture notes in Computer Science 2074(V. Alexendrov, J.Dongarra, B.Julliano, R Renner, C. Tan, eds.), Springer-Verlag , pages 200-210.

[4] S. Parsons, "Current approaches to handling imperfect information in data and knowledge bases," IEEE Transactions on Knowledge and Data Engineering, Vol. 8, 1996, pp. 353-372
[5] Cecilia Temponi, Anthony Shannon, Krassimir Atanassov, Adrian Ban (1999), An idea for an Intuitionistic Fuzzy approach to decision making, Third Int. conf on IFS, Sofia,16-17 October 1999, Notes on IFS, Volume 5 (1999) Number 3, page 6-10.

[6] E. E. Kerre and G. Q. Chen, "An overview of fuzzy data modeling," Fuzziness in Database Management Systems, Physica-Verlag, 1995, pp. 23-41

[7] K T Attanasov (1999) Intuitionistic Fuzzy sets, PhysicaVerlag, Heidelberg, NewYork.

[8] C. Cornelis, E.E Kerre (2002). A Fuzzy Inference methodology based on the Fuzzification of set inclusion. In Innovations in Intelligent Systems(A. Abrahim B.Nath, eds.), studies in Fuzziness and Soft computing series, Springer -Verlag.

[9] Buckles, B.P. and Petry F.E. , A fuzzy representation of data for relational databases, Fuzzy sets and Systems 7(3) (1982) 213-226.

[10] Baoding Liu (2012), Membership functions and operational law of uncertain sets, Fuzzy Optimization and Decision Making, Springer.

[11] F. Herrera, S. Alonso, F. Chiclana and E. HerreraViedma, Computing with words in decision making: foundations, trends and prospects ,From the issue entitled "Special Issue: Computing with Words and Decision Making", Fuzzy Optimization and Decision Making, Springer 